\title{
Análisis de alternativas de producción de semilla vegetativa de Arracacia xanthorrhiza Bancroft en Tolima, Colombia
}

\author{
Analysis of vegetative seed production alternatives of \\ Arracacia xanthorrhiza Bancroft in Tolima, Colombia
}

\author{
Liliana Margarita Atencio Solano' \\ Jorge Enrique Villamil Carvajal ${ }^{2}$ \\ Johanna Paola Garnica Montaña ${ }^{3}$ \\ Antonio María Martínez Reina ${ }^{4}$
}

DOI: https://doi.org/10.19053/01228420.v18.n3.2021.13529

RESUMEN: La propagación de material vegetal en el cultivo de arracacha se realiza comúnmente de forma vegetativa a través de semilla asexual, esta actividad ha permitido su multiplicación y conservación en el tiempo. El material vegetal disponible es de baja calidad, afectando el desarrollo y rendimiento potencial del cultivo y por ende los ingresos del productor. El objetivo de la investigación fue el de analizar comparativamente dos tecnologías de producción de semilla de arracacha: Tecnología local y tecnología Agrosavia. La información para la tecnología local se obtuvo de encuestas aplicadas a los agricultores y la selección se hizo mediante la técnica de muestreo determinístico y para la tecnología Agrosavia a través de registro de datos y costos de producción en lotes de investigación a escala comercial. Se aplicaron estadísticas descriptivas y cálculo de los indicadores de retorno económico para las dos situaciones. Los resultados muestran que el uso de semilla de calidad permite obtener mayor producción de semillas (251.559 unidad $\mathrm{ha}^{-1}$ ) y raíces tuberosas (25.875 $\left.\mathrm{kg} \mathrm{ha}^{-1}\right)$, siendo superior a la tecnología local en $14 \%$ y $28 \%$ respectivamente; de esta forma, el productor de arracacha adquiere mayor eficiencia económica al obtener menor costo unitario por kilo producido y mejor ingreso neto con una tasa de retorno marginal de 316,45 . Los resultados alcanzados son de utilidad para agricultores, empresas y entidades que deseen producir semilla de calidad y apoyen el sistema productivo de arracacha en Colombia.

PAlabras Clave: Semilla Asexual, Calidad, Sistema De Producción, Arracacha, Propagación.
ABSTRACT: The propagation of plant material in the cultivation of arracacha is commonly carried out vegetatively through asexual seed, this activity has allowed its multiplication and conservation over time. The plant material available is of low quality, affecting the development and potential yield of the crop and therefore the income of the producer. The objective of the research was to comparatively analyze two arracacha seed production technologies: Local technology and Agrosavia technology. The information for local technology was obtained from surveys applied to farmers and the selection was made through the deterministic sampling technique, and for the Agrosavia technology data and production costs were recorded in research lots on a commercial scale. Descriptive statistics and calculation of the economic return indicators were applied for the two situations. The results show that the use of quality seed allows obtaining a higher production of seeds $\left(251,559\right.$ unit ha-1) and tuberous roots $\left(25,875 \mathrm{~kg} \mathrm{ha}^{-1}\right)$, being superior to local technology by $14 \%$ and $28 \%$, respectively. Thus, the arracacha producer acquires greater economic efficiency by obtaining a lower unit cost per kilo produced and a better net income with a marginal rate of return of 316.45 . The results achieved are useful for producers, companies, and entities that wish to produce quality seed and support the production system of arracacha in Colombia.

KEY WORDS: asexual seed, quality, production system, arracacha, propagation

FECHA DE RADICACIÓN: 10 de mayo de 2021 FECHA DE APROBACIÓN: 07 de septiembre de 2021

CITAR COMO: Atencio Solano, L. M., Villamil Carvajal, J. E., Garnica Montaña, J. P., Martínez Reina, A. M., \& Ossa Yepes, J. F. Análisis de alternativas de producción de semilla vegetativa de Arracacia xanthorrhiza Bancroft en Tolima, Colombia. Cien. Agri., 18(3): 1-14. https://doi.org/10.19053/01228420.v18. n3.2021.13529

1 M.Sc. Corporación Colombiana de Investigación Agropecuaria - Agrosavia. Turipaná (Colombia). latencio@agrosavia.co. (D) ORCID: https://orcid.org/0000-0001-8425-1621

2 I.A. Corporación Colombiana de Investigación Agropecuaria - Agrosavia. Nataima (Colombia). jvillamil@agrosavia.co. iD ORCID: http://orcid.org/0000-0003-0173-0921

3 M.Sc. Corporación Colombiana de Investigación Agropecuaria - Agrosavia. Nataima (Colombia). jgarnicam@agrosavia.co. (D) ORCID: https://orcid.org/0000-0003-3051-809X

4 Ph.D. Corporación Colombiana de Investigación Agropecuaria - Agrosavia. Turipana (Colombia). amartinezr@agrosavia.co. (D) ORCID: https://orcid.org/0000-0002-9312-842X 


\section{Introducción}

La arracacha (Arracacia xanthorrhiza Bancr.) constituye parte importante de la dieta alimentaria de la población humana, debido a su alto valor nutricional representado en el aporte de proteínas, calcio, fosforo, hierro, carotenos, vitaminas A, B y E (Guerra-Ávila et al., 2012; Pinto-Acero et al., 2019). En Colombia, el cultivo ocupa un renglón importante en el sector primario por su área de siembra y participación en el producto interno bruto (PIB). Durante 2019 se sembró en 14 departamentos en un área total de 9.277 ha, que produjeron $101.604 \mathrm{t}$ con un rendimiento promedio de $8,5 \mathrm{t} \mathrm{ha}^{-1}$, siendo los principales departamentos productores Tolima, Boyacá, Norte de Santander, Cundinamarca y Huila. El municipio con mayor área de siembra es Cajamarca (Tolima) que provee el 53\% de la producción nacional (MinTIC, 2019).

En Tolima se cultivan principalmente tres materiales de arracacha: yema de huevo, cartagenera y amarilla común. Este último, ocupa el 80 - 90\% del área sembrada y se caracteriza por tener alta adaptación agronómica, buena producción de raíces tuberosas, las cuales, son de color amarillo con pigmentación morada en la pulpa y cáscara, condición castigada en el mercado del consumo en fresco (Garnica et al., 2021a; 2021b).

Para fines comerciales la propagación del cultivo de arracacha se desarrolla a través de semilla asexual, denominada comúnmente: semilla, propágulo, hijo, brote o colino (Garnica et al., 2021b). Este tipo de propagación ha sido efectiva en la conservación y producción de cultivos de diferentes especies de raíces y tubérculos. En la reproducción asexual se utiliza un tejido vegetal o sección obtenida de la planta madre con gran potencial de diferenciación celular (Vázquez et al., 1997), dando lugar a una multiplicación rápida y efectiva de plantas, sin variabilidad genética (García, 2002).

El material de propagación de baja calidad afecta el desarrollo, rendimiento potencial de los cultivos (FAO, 2010) y los ingresos del productor. En las zonas productoras de arracacha en Colombia, la semilla vegetativa proviene de los mismos lotes de producción comercial y el proceso de extracción tiene una inadecuada selección (Alvarado et al., 2016), favoreciendo la dispersión de fitopatógenos (Orílio et al., 2013) y afectando el desarrollo del cultivo, producción y calidad de las raíces comerciales (Pinto-Acero et al., 2019).

La anterior problemática se ha evidenciado en diferentes regiones productoras de países como Brasil; razón por la cual, en Brasil y Colombia se han desarrollado modelos de producción de semilla que recomiendan la aplicación de criterios de calidad genética, física, fitosanitaria y fisiológica para garantizar la homogeneidad del material establecido en campo, sanidad y aumento en la producción y calidad de raíces tuberosas (Atencio et al., 2019; Madeira et al., 2017; Sousa-Balbino, 2018). 
Este trabajo tuvo como objetivo hacer un análisis comparativo de las tecnologías de producción de semilla vegetativa de arracacha en la zona productora de Cajamarca (Tolima), considerando el componente agronómico y económico para el sistema de producción local y la tecnología recomendada por Agrosavia en el marco del Plan Nacional de Semillas (2013-2018).

\section{Materiales y Métodos}

\section{A. Localización}

El presente estudio se realizó en el municipio Cajamarca, ubicado sobre la cordillera Central al occidente del departamento de Tolima, en la franja altitudinal de 1.800 a 2.700 m s.n.m., zona de vida de bosque muy húmedo premontano y bosque húmedo montano bajo, que cuenta con una temperatura media de $17,5^{\circ} \mathrm{C}$, humedad relativa promedio de $84,7 \%$ y precipitación anual de 802,1 mm/año (Garnica et al., 2021b).

\section{B. Componente agronómico}

Recolección de información: Se analizaron dos tecnologías de producción de semilla vegetativa de arracacha para el material regional Amarilla común durante los años 2015 y 2018. Se contempló la implementación de los métodos descriptivo y analítico para definir y comparar los dos sistemas.

Tecnología local: Corresponde al manejo tradicional en la zona, la información capturada se obtuvo de agricultores con experiencia en el sistema productivo y que contaban con lotes comerciales establecidos en las veredas Ródano, Tigrera, Cucuana y Leona. Para la captura de información se aplicó la técnica de muestreo determinístico o no probabilístico (Rodríguez, 2005) (Figura 1).

Tecnología Agrosavia: La información se obtuvo de las recomendaciones tecnológicas generadas por la Corporación Colombiana de Investigación Agropecuaria - Agrosavia, a partir de los resultados de ensayos de investigación y pruebas de validación en campo, durante tres ciclos de producción en la vereda Leona cada uno con 12 meses para Amarilla común.

Variables de producción: En la recolección de información se tuvo en cuenta para las dos tecnologías el prendimiento de la semilla (\%), producción de semilla por área (unidad ha-1), producción de semilla por planta (unidad/ planta), semilla enferma (\%), rendimiento de raíces tuberosas (t ha-1) y raíces tuberosas con pigmentación morada (\%). 


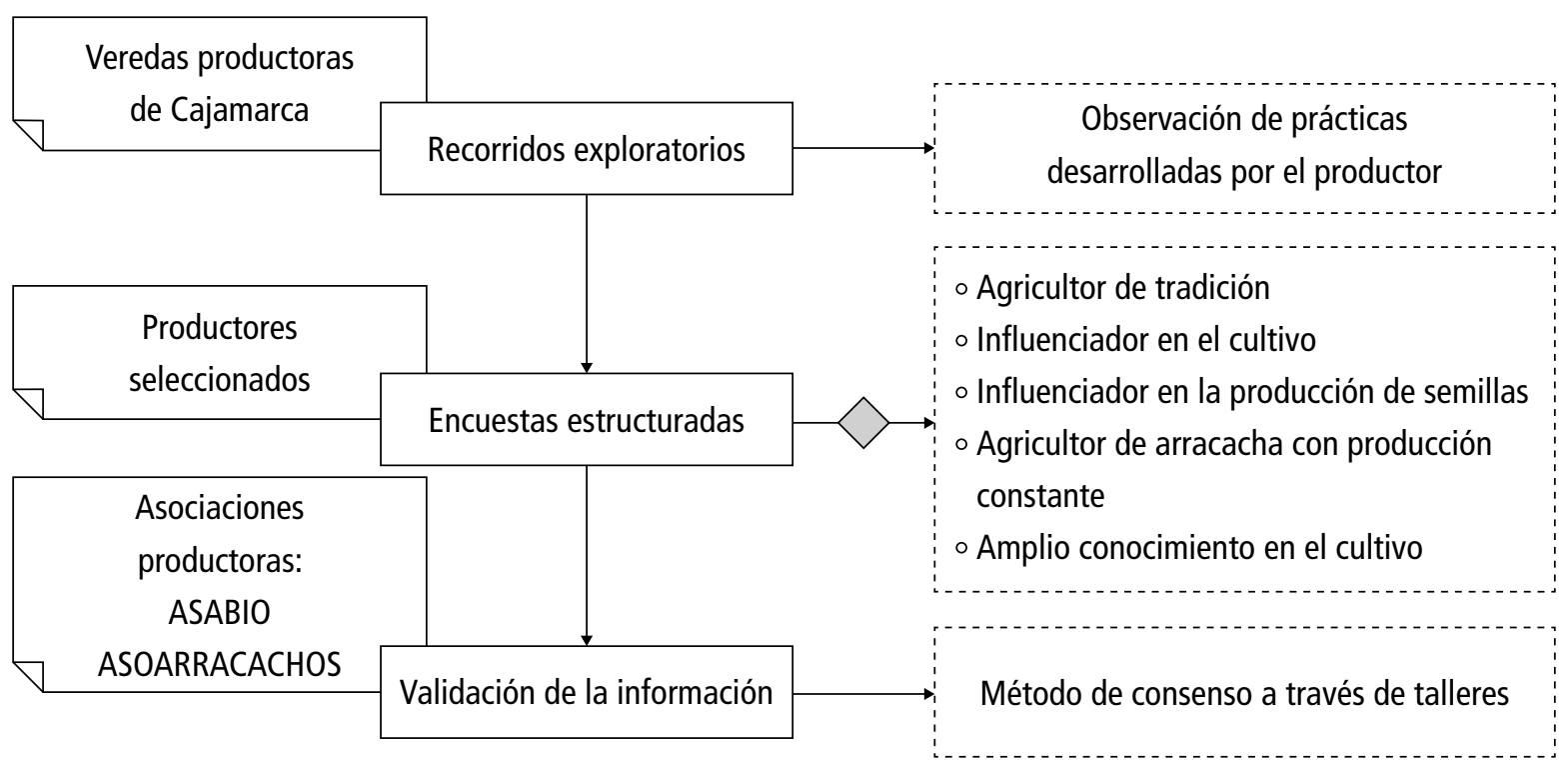

FIGURA 1. Esquema metodológico para la captura de información en la tecnología local de producción de semilla de arracacha en Cajamarca, Tolima.

*El rombo $(\bullet)$ en la figura indican los criterios de selección de productores

\section{Componente económico}

Para las dos tecnologías se estimaron indicadores económicos a partir de la identificación de patrones de costos de producción (Molina et al., 2014), en los cuales, se diferencian los principales componentes: unidad, cantidad, valor unitario (COP) y valor total (COP) de costos directos, indirectos y totales. La mano de obra se valoró de acuerdo con el pago del jornal agrícola en la región y el costo de los insumos a precio de campo (precio que paga el productor por la compra del insumo, más el costo del transporte por llevarlo a la finca), según la metodología (CIMMYT, 1988). Para el análisis de las actividades de manejo del cultivo, se utilizó la metodología empleada por Agreda (1990). Con la información de los rendimientos en las dos tecnologías, se calcularon los costos unitarios y de retorno, como ingresos netos, rentabilidad, punto de equilibrio y eficiencia (Martínez-Reina et al., 2019).

El precio de la raíz tuberosa se calculó teniendo en cuenta el valor promedio mensual en la región (1.298 $\mathrm{COP}_{\text {kilo-1)}}{ }^{-1}$, este valor se multiplicó por el rendimiento (Rdo) y se obtuvo el ingreso bruto (IB). Así, al restar los costos totales de producción (COP) del IB, se calculó el ingreso neto (IN). La rentabilidad se calculó por la relación IN/CT. También se estimó el punto de equilibrio (Peq), que son las cantidades mínimas que se requieren producir para hacer igual los ingresos con los costos (Tabla I). 
TABLA I. Parámetros para la estimación de costos de producción, indicadores económicos y márgenes de comercialización en dos tecnologías de producción de semilla de arracacha en Cajamarca, Tolima.

\begin{tabular}{|c|c|}
\hline Ecuación & Donde: \\
\hline$\sum_{C d=0}^{n} C d=C d 1+C d 2+C d 3+\cdots+C d n$ & $\mathbf{C d}$ : costos directos; $\mathbf{C d}_{1}$ : semillas; $\mathbf{C d}_{2}$ : agroquímicos; $\mathbf{C d}_{3}$ : jornales, etc. \\
\hline$\sum_{C i=0}^{n} C i=C i 1+C i 2+C i 3+\cdots+C i n$ & $\begin{array}{l}\mathrm{Ci}: \text { costos indirectos; } \mathbf{C i}_{1}: \text { alquiler del terreno; } \mathbf{C i}_{2}: \text { costos financieros; } \\
\mathbf{C i}_{3}: \text { otros, etc. }\end{array}$ \\
\hline$C P=C d+C i$ & CP: costos de producción; Cd: costos directos; Ci: costos indirectos. \\
\hline$\sum_{R d o=0}^{n} R d o=P C 1+P C 2+P C 3+\cdots+P C n$ & Rdo: rendimiento; $\mathbf{P C}_{\mathbf{1}}$ : producción total de raíces tuberosas por hectárea. \\
\hline$C U=\frac{C P}{R d o}$ & CU: costos unitarios; CP: costos de producción; Rdo: rendimiento. \\
\hline$I B=R d o * P V$ & $\begin{array}{l}\text { IB: ingresos brutos; Rdo: rendimiento; PV: precio de venta de la producción } \\
\text { de las raíces tuberosas. }\end{array}$ \\
\hline$I N=I B-C P$ & IN: ingresos netos; IB: ingresos brutos; CP: costos de producción. \\
\hline$R T=\frac{I B-C d}{C d} * 100$ & RT: rentabilidad técnica; IB: ingreso bruto; Cd: costos directos. \\
\hline$R N=\frac{I B-C P}{C P} * 100$ & RN: rentabilidad neta; IB: ingreso bruto; Cd: costos de producción. \\
\hline$P e q=\frac{C P}{P V}$ & Peq: punto de equilibrio; Cd: costos de producción; PV: precio de venta. \\
\hline$P P e q=\frac{C P}{I B} * 100$ & $\begin{array}{l}\text { PPeq: porcentaje del punto de equilibrio; Cd: costos de producción; IB: } \\
\text { ingresos brutos. }\end{array}$ \\
\hline$E t=\frac{P V}{C U} * 100$ & Et: eficiencia técnica; PV: precio de venta; CU: costos unitarios. \\
\hline$M B C=\frac{P c-P p}{P c} * 100$ & $\begin{array}{l}\text { MBC: margen bruto de comercialización; Pc: precio del consumidor; } \\
\text { Pp: precio del productor. }\end{array}$ \\
\hline$P D P=100-M B C$ & $\begin{array}{l}\text { PDP: participación directa del productor; MBC: margen bruto de } \\
\text { comercialización. }\end{array}$ \\
\hline$M N C=\frac{M B C-C m}{P C} * 100$ & $\begin{array}{l}\text { MNC: margen neto de comercialización; MBC: margen bruto de } \\
\text { comercialización; } \mathbf{C m :} \text { costos de mercadeo; Pc: precio del consumidor. }\end{array}$ \\
\hline
\end{tabular}

Fuente: Martínez-Reina et al. 2021

Una vez calculados los retornos económicos para las dos tecnologías de producción se hizo el análisis comparativo, considerado como un análisis de presupuesto total para luego aplicar el presupuesto parcial y el análisis marginal (CIMMYT, 1988). El incremento en el ingreso Neto $(\Delta \mathrm{IN})$ y el Incremento en el Costo Variable $(\Delta C V)$ se determinó a partir de los rendimientos de raíces tuberosas $\left(\mathrm{kg} \mathrm{ha}^{-1}\right)$, precio de campo (COP) y los costos variables.

Los rendimientos se multiplicaron por los precios, luego, se restaron los costos que varían y se obtuvo el ingreso neto. Los incrementos en los ingresos netos $(\Delta I N)$ y los incrementos en los costos variables $(\Delta C V)$ se relacionaron para dar lugar a la Tasa de Retorno Marginal que es la posibilidad de recuperar los costos adicionales por la tecnología. 


\section{Resultados y Discusión}

\section{A. Componente agronómico}

A través del estudio se logró identificar para las dos tecnologías analizadas tres etapas: identificación de lote y semilla, manejo agronómico y cosecha de las raíces tuberosas. Definidas con actividades particulares dependiendo de la tecnología de producción (Figura 2).

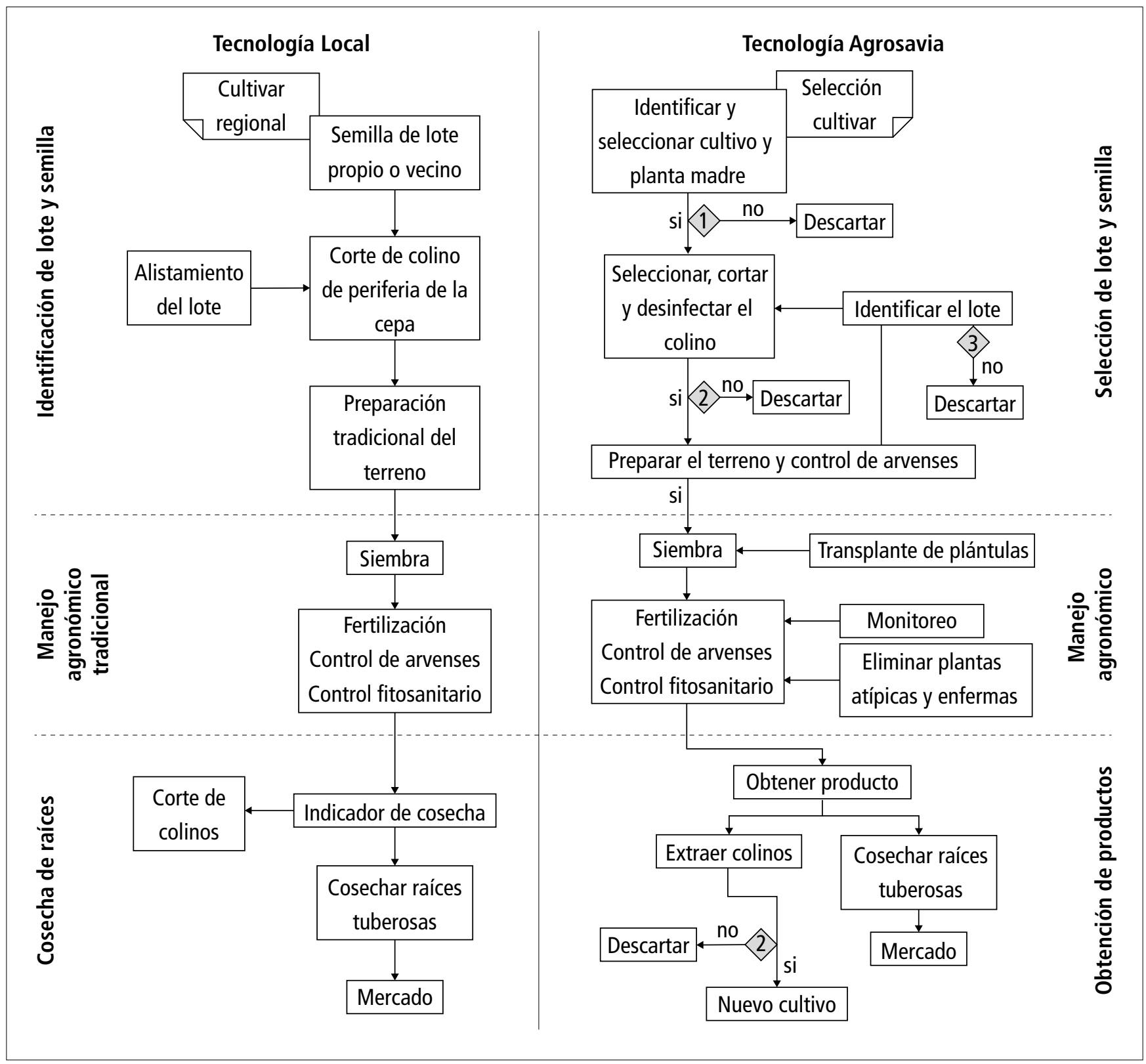

FIGURA 2. Esquema para dos tecnologías de producción de semilla de arracacha en Cajamarca, Tolima.

* Los rombos $(\bullet)$ en la figura indican que se debe tomar una decisión con base en los criterios asociado al factor. 1. ¿La planta madre cumple con criterios de calidad?; 2. ¿Los semillas cumplen con criterios de calidad?; 3. ¿El lote cumple con los criterios para la producción de semilla de arracacha? 


\section{Tecnología local}

Identificación de lote y semilla: De acuerdo con la información suministrada por los agricultores encuestados, la selección del lote para siembra se hace con base en su experiencia empírica, sin tener en cuenta la incidencia de plagas y enfermedades del suelo, rotación de cultivos y descanso del terreno, que coincide, con lo expuesto por Alvarado et al. (2016). Entre tanto, la producción de semilla se deriva de prácticas tradicionales, factor que es común en otras zonas productoras de arracacha (Alvarado \& Ochoa, 2010a; Alvarado \& Pita, 2016).

La semilla se obtiene de plantas que presentan la parte aérea visualmente sana, ubicadas en lotes con relieve ondulado a quebrado, próximas a la cosecha de raíces tuberosas, y su alistamiento no contempla la extracción de la planta completa, por lo cual, no es posible conocer el estado fitosanitario de los órganos subterráneos como cepa y raíces tuberosas, favoreciendo la dispersión de fitopatógenos (Orílio et al., 2013). Al final el productor obtiene semilla sin homogeneidad en edad, procedencia y calidad.

Manejo agronómico: El sistema productivo no cuenta con asistencia técnica especializada y gran parte del conocimiento se transmite de generación en generación. Las labores agrícolas incluyen prácticas de fertilización sin análisis de suelos y aplicaciones continuas en drench, manejo fitosanitario sin monitoreo de poblaciones de dosel y suelo, control manual malezas y aplicación de herbicidas en épocas cercanas a cosecha como estrategia para reducir costos de producción. Estas prácticas agronómicas coinciden con lo documentado en los trabajos realizados en el departamento de Boyacá por Alvarado \& Ochoa (2010b) y Muñoz et al. (2015).

Cosecha: En el material amarilla común la cosecha de las raíces tuberosas se realiza a los 12 meses para la franja altitudinal de 1.800 a 2.200 m s.n.m., y en altitudes superiores después de los 13 meses de edad. En esta tecnología las raíces tuberosas son el principal producto y la semilla vegetativa es considerada un subproducto sin valor comercial, razón por la cual, esta se adquiere por intercambio u obsequio entre agricultores en un proceso de movilización local (flujo espontáneo) sin aplicar criterios de selección de calidad genética, física, fisiológica y sanitaria.

\section{Tecnología Agrosavia}

Selección del lote y semilla: Se recomienda la selección de lotes con pendiente superior al $30 \%$, suelos profundos con textura franco-arenosa y buen drenaje, acceso a agua, ausencia de arvenses limitantes en la producción como Artemisia spp., baja presión de insectos-plaga como chiza y nemátodos identificados mediante diagnostico en campo y análisis microbiológico del suelo, lotes con descanso y rotación de cultivos, evitando siembras en areas 
establecidas durante los últimos años con tomate y repollo por compartir fitopatógenos del suelo.

Para la obtención de semilla asexual se siguen los criterios de calidad expuestos en la Tabla II, los cuales, concuerdan con la investigación en modelos de producción de semilla vegetativa en Brasil por parte de Madeira et al. (2017) y Sousa-Balbino (2018).

TABLA II. Criterios de calidad para la selección de plantas y semilla en el material de arracacha amarilla común, Cajamarca, Tolima.

\begin{tabular}{|c|c|c|}
\hline Criterios & Plantas & Semilla asexual \\
\hline $\begin{array}{l}\text { Calidad } \\
\text { genética }\end{array}$ & $\begin{array}{l}\text { - Características propias del material amarilla común } \\
\text { - Características homogéneas en el cultivo, sin mezcla de } \\
\text { genotipos }\end{array}$ & $\begin{array}{l}\text { Debe corresponder al } \\
\text { genotipo a sembrar }\end{array}$ \\
\hline $\begin{array}{l}\text { Calidad } \\
\text { fisiológica }\end{array}$ & $\begin{array}{l}\text { - Vigorosas entre } 10 \text { y } 14 \text { meses de edad } \\
\text { - Sin deficiencias nutricionales } \\
\text { - Sin afectación por déficit hídrico }\end{array}$ & $\begin{array}{l}\text { Base redonda } \\
\text { seleccionado de la } \\
\text { periferia de la cepa }\end{array}$ \\
\hline Calidad física & $\begin{array}{l}\text { - Altura mayor a } 50 \mathrm{~cm} \\
\text { - Peso de raíces tuberosas entre } 2 \text { y 2,5 kg } \\
\text { - Plantas con } 30 \text { - } 45 \text { peciolos } \\
\text { - Plantas con } 10 \text { - } 15 \text { semillas desarrolladas } \\
\text { - Tejidos internos de semillas y raíces tuberosas sin } \\
\text { alteraciones en la coloración } \\
\text { - Sin daños mecánicos } \\
\text { - Raíces tuberosas completamente amarillas }\end{array}$ & $\begin{array}{l}\text { - Características } \\
\text { homogéneas } \\
\text { - 2-3 anillos diferenciados } \\
\text { de la cepa y } 5-7 \mathrm{~cm} \text { de } \\
\text { longitud } \\
\text { - Ausencia de } \\
\text { pigmentaciones violetas } \\
\text { en los tejidos internos }\end{array}$ \\
\hline $\begin{array}{l}\text { Calidad } \\
\text { fitosanitaria }\end{array}$ & $\begin{array}{l}\text { - Las manchas foliares ocasionadas por los hongos } \\
\text { Alternaria sp., Cercospora sp. y Colletotrichum sp. no } \\
\text { deben superar el } 5 \% \text { de severidad. } \\
\text { - Plantas libres de insectos-plaga, gusano viringo, } \\
\text { babosas, trips, áfidos, ácaros y enfermedades del suelo } \\
\text { asociadas a hongos, bacterias y/o nemátodos } \\
\text { - Cepa sin alteraciones en tejidos (pudrición acuosa o } \\
\text { pigmentaciones marrón) }\end{array}$ & $\begin{array}{l}\text { Ausencia de daños } \\
\text { asociados a plagas y/o } \\
\text { enfermedades. }\end{array}$ \\
\hline
\end{tabular}

Fuente: Elaboración propia

Manejo agronómico: El establecimiento prioriza la semilla pre-enraizada en semilleros, previa desinfección con hipoclorito de sodio al 5\% y/o yodo agrícola al 2\%, para reducir perdidas de material y tener homogeneidad en campo. La nutrición del cultivo se hace con base en análisis de suelos, el manejo fitosanitario de acuerdo con monitoreos quincenales y mensuales, que incluye la eliminación de plantas atípicas o enfermas y el control de arvenses con el uso de herbicidas en las primeras etapas de crecimiento y limpia manual a partir del llenado de raíces. Según Garnica et al. (2021b) y Sousa-Balbino (2018), el manejo oportuno del cultivo siguiendo las recomendaciones técnicas mejoran el rendimiento y aseguran semilla de calidad. 
Obtención de productos: El cultivo se direcciona con dos finalidades: semiIla y raíces tuberosas de calidad. Se logra semilla con mejor prendimiento, mayor número por unidad de área y ausencia de problemas fitosanitarios, y se obtiene mayor producción de raíces tuberosas con menor porcentaje de pigmentación morada (Tabla III) (Atencio et al., 2019). Para promover la calidad de los dos productos, se deben programar las actividades de alistamiento del producto (semilla y raíces tuberosas) con antelación a la cosecha considerando la ubicación altitudinal del lote.

\section{Variables de producción}

La metodología aplicada en las dos tecnologías permitió determinar indicadores de producción a partir de la cuales se hizo un análisis comparativo (Tabla III).

TABLA III. Análisis comparativo de variables de producción para dos tecnológicas de obtención de semilla de arracacha en Cajamarca, Tolima, 2020.

\begin{tabular}{|l|c|c|}
\hline Variables & Tecnología local & Tecnología Agrosavia \\
\hline Prendimiento (\%) & 90 & 95 \\
\hline Producción de semilla (unidad ha-1) & 216.000 & 251.559 \\
\hline Producción de semillas (unidad/ planta) & 10 & 22 \\
\hline Semillas enfermos (\%) & 5 & 0 \\
\hline Producción raíces tuberosas $\left(\mathrm{kg} \mathrm{ha}^{-1}\right.$ ) & 18.625 & 25.875 \\
\hline Pigmentación morada de raíces tuberosas (\%) & 7 & 4 \\
\hline
\end{tabular}

La tecnología Agrosavia mostró mayor producción de semillas por unidad de área (251.559 semillas ha $\left.{ }^{-1}\right)$, superando en 14\% (35.559 semillas) a la tecnología local, lo que permite el establecimiento de 12,6 ha en promedio y garantiza mayor prendimiento (95\%), población de plantas uniformes, mayor rendimiento y menor pigmentación morada en raíces tuberosas (4\%).

De igual forma, la tecnología Agrosavia permitió obtener una producción total de raíces tuberosas de $26.953 \mathrm{~kg} \mathrm{ha}^{-1}$, de las cuales, el 96\% (25.875 kg $\left.\mathrm{ha}^{-1}\right)$ corresponden a raíces comerciales y el $4 \%\left(1.078 \mathrm{~kg} \mathrm{ha}^{-1}\right)$ restante a no comerciales que se descartan por presencia de pigmentación morada. En comparación con la tecnología local que logró una producción total de raíces tuberosas de $20.027 \mathrm{~kg} \mathrm{ha}^{-1}$, donde, el 93\% (18.625 $\left.\mathrm{kg} \mathrm{ha}^{-1}\right)$ son comerciales y el $7 \%\left(1.401,9 \mathrm{~kg} \mathrm{ha}^{-1}\right)$ se descartan por pigmentación morada.

La discusión de las tecnologías tuvo un enfoque en la producción de semilla de arracacha, sin embargo, comercialmente el producto principal es la raíz tuberosa. Bajo esta condición, es importante resaltar que la semilla actualmente no tiene un valor comercial, pese a ello, con la aplicación de recomendaciones y el surgimiento de expertos para la producción de semilla en un 
futuro, podría adquirir valor económico que representaría a su vez un valor agregado para el agricultor, como sucede en Brasil, donde generalmente la siembra se realiza a partir de semilla seleccionada con criterios de calidad, lo que ha permitido altos rendimientos en producción (Madeira et al., 2017; Morillo et al., 2020).

\section{B. Componente económico}

Con base en los indicadores de producción, en la tabla IV se presenta el análisis económico para las dos tecnologías, basado en los precios (COP) del año 2020 para raíces tuberosas en fresco y una densidad poblacional de 16.666 plantas ha ${ }^{-1}$.

TABLA IV. Análisis comparativo de costos y retornos económicos de dos tecnologías de producción de semilla de arracacha en Cajamarca, Tolima, 2020.

\begin{tabular}{|l|c|c|}
\hline Indicador & Tecnología local & Tecnología Agrosavia \\
\hline Labores (COP) & 8.135 .000 & 9.472 .300 \\
\hline Insumos (COP) & 1.435 .450 & 2.210 .034 \\
\hline Herramientas (COP) & 898.400 & 898.400 \\
\hline Subtotal Costos Directos (COP) & 10.468 .850 & 12.580 .734 \\
\hline Costos Indirectos (COP) & 2.032 .820 & 2.180 .651 \\
\hline Costos Totales (COP) & 12.501 .670 & 14.761 .385 \\
\hline Rendimiento (kg ha-1) & 18.625 & 25.875 \\
\hline Costo unitario por kg (COP) & 671 & 570 \\
\hline Precio de venta por kg (COP) & 1.298 & 1.298 \\
\hline Ingreso Bruto (COP) & 24.175 .250 & 33.585 .750 \\
\hline Ingreso Neto (COP) & 11.673 .581 & 18.824 .365 \\
\hline Rentabilidad (\%) & 93 & 128 \\
\hline Punto de equilibrio (kg) & 9.631 & 11.372 \\
\hline Porcentaje del punto de equilibrio & 52 & 44 \\
\hline Eficiencia & 1,9 & 2,3 \\
\hline
\end{tabular}

Fuente: Elaboración propia con base en Garnica et al. (2021b)

Los resultados muestran que las dos tecnologías son eficientes en la producción comercial de raíces tuberosas. La tecnología Agrosavia permite obtener rendimientos de raíces tuberosas más altos, con una diferencia de $7.250 \mathrm{~kg}$ $\mathrm{ha}^{-1}$, con relación a la tecnología local. A esto se suma, que los costos unitarios en la tecnología Agrosavia son más bajos con una diferencia de 101 COP $\mathrm{kg}^{-1}$, lo cual permite que el ingreso sea mayor (18.824.365 COP) logrando una diferencia favorable de 7.150.784 $\mathrm{COP} \mathrm{ha}^{-1}$. El punto de equilibrio es más alto en la tecnología local requiriendo de un $52 \%$ de la producción para recuperar 
los costos de producción, con respecto a un 44\% de la tecnología Agrosavia que representa ventajas económicas para el productor.

El análisis marginal en algunas situaciones se hace cuando se evalúan tecnologías nuevas para proceder con una recomendación (CIMMYT, 1988 ). En este caso se tomaron los cambios en el ingreso neto y en el costo variable, es de anotar, que sólo se hizo sobre los componentes que cambiaron y se relacionaron con los cambios en el beneficio neto. Los cambios en el beneficio neto fueron mayores al costo variable, mientras que, el costo variable cambió en 2.259.716 COP ha ha $^{-1}$, los beneficios netos lo hicieron en 7.150.784 COP y la tasa de retorno marginal fue de 316,45. Esto indica que, por cada unidad monetaria invertida, se recupera y genera adicionalmente 2,16 COP, lo que incrementa el ingreso neto con respecto a los costos de inversión y hace viable la tecnología Agrosavia (Tabla V).

TABLA V. Análisis marginal para dos alternativas tecnológicas de producción de arracacha en Cajamarca Tolima 2020.

\begin{tabular}{|l|c|c|c|c|c|}
\hline Indicador & IN & CV & AIN & ACV & TRM \\
\hline Tecnología local & 11.673 .581 & 0 & 7.150 .784 & 2.259 .716 & 316,45 \\
\hline Tecnología Agrosavia & 18.824 .365 & 2.259 .716 & & & \\
\hline
\end{tabular}

IN = Ingreso Neto, $C V=$ Costos Variable, $\Delta \mathrm{IN}=$ Incremento en el ingreso Neto, $\Delta \mathrm{CV}=$ Incremento en el Costo Variable. Fuente: Elaboración propia

No obstante, la tecnología Agrosavia incurre en mayores costos, los cuales, se ven compensados con mejores rendimientos evidenciados en un mayor ingreso neto, es decir, cada vez que el agricultor incorpora esta tecnología a la producción local los ingresos netos aumentan, lo que sugiere hacer estos cambios al sistema productivo de arracacha en la región.

\section{Conclusiones}

El cultivo de arracacha en Cajamarca (Tolima) presenta un sistema de producción de semilla informal desarrollado en tres etapas: identificación de lote y semilla, manejo agronómico tradicional y cosecha de las raíces tuberosas, en donde, existe un enfoque cultural basado en la movilización espontanea de semilla.

La producción de semilla de calidad requiere la selección de lotes con baja presión de plagas y enfermedades del suelo e historial conocido, selección de semilla a partir de plantas madre que reúnan criterios de calidad genética, física, fisiológica y fitosanitaria, manejo agronómico con enfoque agroecológico y obtención de dos productos (raíces tuberosas y semilla de calidad). 
La planta madre es el insumo principal para la producción de semilla de calidad, los principales criterios que definen la selección en el cv. amarilla común son: plantas vigorosas libres de insectos-plaga y enfermedades del dosel y suelo, sin deficiencias nutricionales y afectación por déficit hídrico, raíces completamente amarillas y sin daño por nematodos.

El uso de semilla de calidad permite la obtención de mayor producción de semillas ha-1 y raíces tuberosas, con menor pigmentación morada, favoreciendo los ingresos del agricultor.

\section{Agradecimientos}

Al Programa Nacional de Semillas, un acuerdo interinstitucional entre la Corporación Colombiana de Investigación Agropecuaria (AGROSAVIA), el Instituto Colombiano Agropecuario (ICA) y el Servicio Nacional de Aprendizaje (SENA), por su apoyo para el desarrollo y cumplimiento del proyecto Consolidación del núcleo de producción de semilla seleccionada de arracacha de variedades mejoradas y regionales en el cañón del Anaime (Tolima) y otras regiones productoras. A la Asociación de Productores de Semillas Andinas (ASABIO), la Asociación de Arracacheros de Cajamarca y Anaime (ASOARRACACHOS) y a Juan Felipe Ossa Yepez por la traducción de los apartados del manuscrito.

\section{Referencias}

Agreda, V. (1991). Metodología para el análisis económico. In B. Quijandria \& M. E. Ruiz (Eds.), Aspectos metodológicos del análisis social en el enfoque de sistemas de producción (pp. 93-124). Red de Investigación en Sistemas de Producción Animal en Latinoamérica-RISPAL Centro de Estudios y de Desarrollo Agrario del Perú.https://repositorio.iica.int/bitstream/handle/11324/8853/ BVE20037866e. pdf? sequence $=1$ \&isAllowed=y

Alvarado, Á.E. \& Ochoa, L.E. (2010a). Tecnologías locales de producción de arracacha (Arracacia xanthorrhiza Bancroft) en el municipio de Boyacá, departamento de Boyacá. Rev. U.D.C.A Act. \& Div. Cient, 13(1), 125-133. https://doi. org/10.1371/journal.pone.0152157

Alvarado, Á.E. \& Ochoa, L.E. (2010b). Cultivo de arracacha (Arracacia Xanthorrhiza Bancroft) en los municipios de Turmequé y Boyacá. Universidad pedagógica y Tecnológica de Colombia. https://uptc.metalibros.org/index.php/ editorial-uptc/catalog/download/99/126/1845-1?inline=1

Alvarado Gaona, A.E., Muñoz, A.L., \& Adame, O.F. (2016). Una aproximación al desarrollo rural, caso el cultivo de arracacha, en el departamento de Boyacá. Ingeniería y Región, 16(2), 57. https://doi.org/10.25054/22161325.1299

Alvarado Gaona, Á. E., \& Pita Morales, L. A. (2016). Sistema agroalimentario del cultivo de arracacha (Arracacia xanthorrhiza Bancroft), desde la multifuncionalidad, municipio de Boyacá (Boyacá). Conexión Agropecuaria JDC, 6(2), 19-31. https://jdc.edu.co/revistas/index.php/conexagro/article/view/563/573 
Atencio Solano, L. M., Garnica Montaña, J. P., Vargas Berdugo, A. M., Villamil Carvajal, J. E., \& Cañar Serna, D. Y. (2019). Manual técnico para la producción de semillas de arracacha bajo condiciones agroecológicas del municipio de Cajamarca, Tolima. Corporación Colombiana de Investigación Agropecuaria - AGROSAVIA. https://doi.org/10.21930/agrosavia.manual-19

Centro Internacional de Mejoramiento de Maíz y Trigo - CIMMYT. (1988). La formulación de recomendaciones a partir de datos agronómicos: Un manual metodológico de evaluación económica. México D.F. - México. 79 pp. https://repository. cimmyt.org/xmlui/bitstream/handle/10883/1063/9031.pdf

García Forero, D. (2002). Protocolo para la propagación vegetativa de las especies Tabebuia rosea (bertol) DC. y Cordia alliodora (Ruíz \& Pavón) Oken por medio de propagulos. Colombia Forestal, 7(15), 109-117.

https://doi.org/https://doi.org/10.14483/2256201X.3311

Garnica Montaña, J. P., Villamil Carvajal, J. E., Atencio Solano, L. M., \& Jaramillo-Barrios, C. I. (2021a). Caracterización fenotípica de tres cultivares regionales de arracacha (Arracacia xanthorrhiza Bancroft) en Tolima, Colombia. Ciencia y Agricultura, 18(2), 1-20. https://doi.org/10.19053/01228420.v18.n1.2021.12041

Garnica Montaña, J. P., Villamil Carvajal, J. E., Vargas Berdugo, Á. M., Rodríguez Rodríguez, O. J., \& Atencio Solano, L. M. (2021b). Modelo productivo de arracacha (Arracacia xanthorrhiza Bancr.) Agrosavia la 22 para la región andina de Colombia. Corporación Colombiana de Investigación Agropecuaria AGROSAVIA. https://doi.org/10.21930/agrosavia.nbook.7404500

Guerra-Ávila, D., González-Melo, F., \& Alvarado-Gaona, Á. (2012). Producción y residuos en dos materiales comerciales de arracacha (Arracacia xhanthorriza Bancr.) en Boyacá (Boyacá). Ciencia y Agricultura, 9(2), 77-86. https://www. metarevistas.org/Record/oai:ojs:revistas.uptc.edu.co:articleojs-2818

Madeira, N., Ferreira, A., Da Silva, G., Borges, J., Borges, R., Michereff, M., Pires, N., Moreira, S., Ribeiro, G., \& Cássia, R. (2017). Proposição de um Sistema de Produção de Mudas de Mandioquinha-salsa. Circular Técnica, 161. https://www. researchgate.net/publication/321907164_Proposicao_de_um_Sistema_de_Producao_de_Mudas_de_Mandioquinha-salsa

Martínez-Reina, A. M., Tordecilla-Zumaqué, L., Cordero-Cordero, C., \& Grandett-Martínez, L. (2019). Entorno tecnológico y socioeconómico de la habichuela larga en el Caribe Húmedo de Colombia. Ciencia y Agricultura, 16(2), 7-24. https:// doi.org/10.19053/01228420.v16.n2.2019.9114

Martínez-Reina, A. M., Grandett-Martínez, L., Tordecilla-Zumaqué, L., Rodríguez-Pinto, M., Cordero-Cordero, C. \& Tofiño-Rivera, A. (2021). Análisis tecnológico y socioeconómico del sistema de producción local del frijol rosado Zaragoza (Phaseolus vulgaris L.) en el Caribe de Colombia. Revista colombiana de Ciencias Hortícolas, 15(1), 1-21. https://doi.org/10.17584/rcch.2021v15i1.11520

Ministerio de Tecnologías de la Información y las Comunicaciones - MinTIC. (2019). Evaluaciones Agropecuarias Municipales - EVA. Datos abiertos.https://www.datos.gov.co/Agricultura-y-Desarrollo-Rural/EvaluacionesAgropecuarias-Municipales-EVA/2pnw-mmge/data

Molina, N. A., Acuña, L. E., \& Marmelicz, L. A. (2014). Costo de producción y rentabilidad del ananá en la provincia de Misiones. Publicación EEA INTA Bella Vista. 
Serie Técnica No 47. Argentina. https://inta.gob.ar/sites/default/files/scripttmp-inta_-_s_t_n_47_-_costo_de_produccin_y_rentabilidad.pdf

Muñoz, A.L., Alvarado, A.E. \& Almanza, P.J. (2015). Caracterización preliminar del cultivo de arracacha Arracacia xanthorrhiza Bancroft en el departamento de Boyacá. Revista Ciencias Agrícolas, 32(1), 3-11. http://dx.doi.org/10.22267/ rcia.153201.20

Organización de las Naciones Unidas para la Alimentación y la Agricultura - FAO. (2010). Quality declared planting material. Protocols and standards for vegetatively propagated crops. In FAO Plant Production and Protection Paper 195. 126 pp. http://www.fao.org/3/a-i1 195e.pdf

Orílio, A. F., Lucinda, N., Dusi, A. N., Nagata, T., \& Inoue-Nagata, A. K. (2013). Complete genome sequence of arracacha mottle virus. Archives of Virology, 158(1), 291-295. https://doi.org/10.1007/s00705-012-1473-x

Pinto-Acero, Y. L., Alvarado-Gaona, Á. E., Burgos-Ávila, Y. E., Balaguera-lópez, H. E., \& Ramírez-Gonzáñez, S. I. (2019). Caracterización de tres genotipos de Arracacia xanthorrhiza Bancroft mediante parámetros morfológicos y de color. Revista Colombiana de Ciencias Hortícolas, 13(3), 426-434. https://doi.org/https:// doi.org/10.17584/rcch.2019v13i3.8948

Sousa-Balbino, J.M. (2018). Cultura da batata-baroa (mandioquinha-salsa): práticas da produção à pós-colheita (Sousa-Balbino José M. Sousa Balbino (ed.)). Instituto Capixaba de Pesquisa, Assistência Técnica e Extensão Rural. Brasil. https:// biblioteca.incaper.es.gov.br/digital/handle/123456789/3382

Rodríguez Osuna, J. (2005). Métodos de muestreos. Centro de Investigaciones Sociológicas. Madrid.

Vázquez Yanes, C., Orozco, A., Rojas, M., Sánchez, M. E., \& Cervantes, V. (1997). La reproducción de las plantas: Semillas y meristemos. Fondo de Cultura Económica. México D.F. - México. http://bibliotecadigital.ilce.edu.mx/sites/ciencia/ volumen3/ciencia3/157/htm/lcpt157.htm 